\title{
Leveraging next-generation phenotyping and pan-omics to identify novel biomarkers and pharmaceutical targets for cardiovascular disease
}

Keywords: atherosclerosis $\bullet$ bioinformatics $\bullet$ cardiovascular CT $\bullet$ coronary artery disease
- gene expression $\bullet$ genomics proteomics $\bullet$ lipidomics $\bullet$ metabolomics $\bullet$ systems biology

At 18 weeks of pregnancy, a 28-year-old female with a family history of deep venous thrombosis and breast cancer undergoes peripheral blood draw, and whole-genome sequencing (WGS) of the fetus is performed on fetal DNA extracted from maternal blood. The 29-year-old father has a family history of premature coronary artery disease (CAD), Type 2 diabetes and age-related macular degeneration (AMD). Fetal WGS of a male fetus reveals susceptibility loci for prostate cancer, hypothyroidism, Type 2 diabetes, dyslipidemia related to PCSK9 abnormality and AMD. At birth, height, weight and Apgar scores are normal. At age 18 years, prostate biomarkers and imaging, thyroid biomarkers and imaging, and eye exam are normal; an apoB vaccine is administered for PCSK9related dyslipidemia. At age 40 years, prostate and thyroid biomarkers and imaging remains normal; lipids/lipoproteins and anti-apoB titers are normal. At age 43 years, during an upper respiratory infection, a toilet-based sensor detects hyperglycemia during urination an hour after dinner; office-based blood tests reveal hepatic insulin resistance and hyperglycemia. Specific therapy is administered to reverse hepatic insulin resistance; within 12 weeks, insulin sensitivity and blood glucose levels return to normal; noninvasive cardiovascular imaging reveals no evidence of atherosclerosis. At age 56 years, annual targeted pan-omic evaluation detects abnormal prostate signature; targeted imaging reveals abnormal molecular activity in the epithelial cells in the prostate. Targeted infusion therapy reverses the abnormality in 8 weeks. At age 83 years, automated bathroom mirrorbased sensor indicates early signs of AMD; induced pluripotent stem cells are injected peripherally and home to the retina to replace degenerated macular cells. At age 84 years, the patient remarries and lives another 9 years.

The above hypothetical case presentation sums up the goal of the future of medicine: to ensure long, productive and healthy life based on the understanding of individual genetic susceptibilities, and serial, targeted evaluation of blood-based biomarkers and imaging throughout life to monitor health and disease based on specific phenotypes.

Unfortunately, there are many major hindrances to such an approach today. First and foremost, one of the major challenges of today's medicine is that it is population-based and probabilistic, as opposed to being individualized. Current clinical practice guidelines are based in population medicine, and deal with probabilities, with no regard to actual individual patient biology. It has been shown that a large proportion of patients with low and intermediate Framingham Risk Score (FRS), the most common global risk stratification instrument in the USA, have atherosclerotic $\mathrm{CAD}$ as detected by cardiovascular computed tomography (CT) [1]. Furthermore, the FRS underestimates the incidence of coronary events in four out of ten subjects, as shown in the Multi-Ethnic Study of Atherosclerosis [2]. Finally, over $60 \%$ of patients presenting with an acute coronary event have low FRS.

Another, more recent case in point are the new guidelines for the management of cholesterol disorders, released by the American

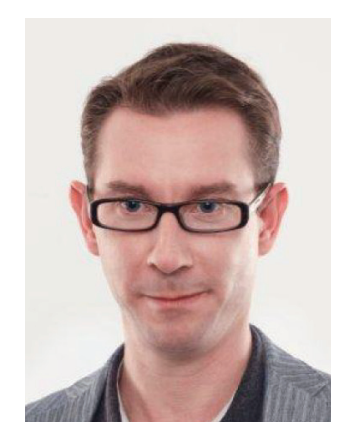

Szilard Voros

Global Genomics Group, LLC, 737 North, 5th Street, Richmond, VA 23219, USA and

Stony Brook University, Stony Brook, NY, USA

Tel.: +1 4043681554

Fax: +1 8048843889

szilard.voros@globalgenomicsgroup.com 
College of Cardiology and the American Heart Association in 2013 [3]. This document and approach, in my view, represents an unfortunate, but significant step back in the management of individual abnormalities in cholesterol metabolism and cardiovascular disease. An exaggerated and rigid interpretation of previous randomized controlled trials of elevated cholesterol resulted in a document, which may be interpreted with the following basic premise, from the perspective of a biologist: cardiovascular disease is caused by statin deficiency and statin replacement results in improved cardiovascular outcomes. This approach collides head on with the basic biological premise, upon which these very randomized controlled trials were designed: elevated low-density lipoprotein (LDL) cholesterol is causative in atherosclerotic CAD, and lowering LDL cholesterol, for example, with bile acid sequestrants, fibrates, niacin and statins, results in improved cardiovascular outcomes.

\section{“...all previous coronary artery disease- related genome-wide association studies have utilized genome-wide genotyping by tagging a few hundred thousand to up to 2 million tag SNPs, rather than whole-genome sequencing on a large scale."}

Another major challenge is a practical one; there is a major disconnect and significant time lag between cutting-edge scientific discoveries, clinical practice guidelines, appropriate use criteria, and Current Procedural Terminology codes and reimbursement. It is quite thought provoking that current reimbursement structures, including the Congressional Business Office, are based on models of treatment of disease, as opposed to early detection, prevention and early intervention. In fact, the Congressional Business Office openly scores prevention as a cost.

How can we overcome at least the scientific challenges that lie ahead? In my view, we have to move away from the traditional models of hypothesis-driven discovery on the basis of painstakingly collected biological evidence in the laboratory over many decades toward a hypothesis-free, unbiased discovery paradigm anchored in three fundamental pillars:

- Precision phenotyping with tools such as advanced, noninvasive imaging;

- Next-generation, best-in-class pan-omics using whole-genome and transcriptome sequencing, unbiased proteomics and metabolomics;

- Systems biology-driven bioinformatics.
Blood- and tissue-based discovery of pan-omic signatures are critical for the assembly of comprehensive, multidimensional networks of health and disease, and perturbation of such networks can be understood in different disease states [4]. Centrally located molecules in such comprehensive networks may emerge as putative diagnostic and prognostic biomarkers, as well as therapeutic targets of different disease states.

One example of such a comprehensive approach is the currently ongoing prospective, international, multicenter study of atherosclerotic CAD, the GLOBAL study [5]. GLOBAL is currently one of the most ambitious, largest pan-omic studies of any disease state, and is one of the largest prospective studies of WGS. The study is recruiting subjects referred for cardiovascular CT examination, and CT is utilized as the first pillar, for precision phenotyping, based on previously validated methods [6-9]. As the second pillar, all patients undergo detailed, comprehensive panomics from peripheral blood including WGS, DNA methylation, evaluation of copy number variants, whole-blood-based transcriptome sequencing, unbiased, mass spectrometry-based proteomics, metabolomics and lipidomics. As the third fundamental pillar, a systems biology-driven bioinformatics approach is utilized to synthesize the approximately 22 trillion data points collected in the study to create novel, comprehensive biological networks of atherosclerosis for the discovery of centrally located key molecules in CAD.

In the past, several studies have evaluated genetic underpinnings of $\mathrm{CAD}$, using different approaches. From a phenotyping perspective, previous CADrelated genomic studies utilized the clinical phenotype of history of myocardial infarction, or invasive coronary angiography. However, the use of myocardial infarction as a phenotype is limited by the fact that it combines different biologies, including that of atherosclerosis, plaque rupture and thrombosis. From a genotyping perspective, all previous CADrelated genome-wide association studies have utilized genome-wide genotyping by tagging a few hundred thousand to up to 2 million tag SNPs, rather than WGS on a large scale. Nevertheless, as of 2013, approximately 46 genetic loci have been shown to be associated with CAD [10].

Several previous studies also evaluated bloodbased gene expression signatures in CAD patients. The PREDICT investigators utilized RNA microarrays extracted from circulating mononuclear cells, using invasive coronary angiography-defined 50\% luminal diameter stenosis as definition for CAD. An algorithm containing age, sex and quantitative 
PCR-quantified expression levels of 23 genes was validated to have good discrimination and reclassification for the diagnosis of CAD [11,12]. In addition, the same algorithm has been validated in the COMPASS study by our laboratory, using CT angiography as the predominant phenotyping tool [13]. In addition, this score has also been shown to predict outcomes [12], as well as to be associated with plaque composition by intravascular ultrasound [14] and CT angiography [15].

Blood-based proteomic associations of CAD have also been described previously, mostly using clinical event-based case definitions from population-based cohorts [16]. However, no previous large studies of unbiased proteomics have utilized advanced imaging as a phenotyping tool for atherosclerosis.

Some authors suggested that metabolomics may be the most practical way of assessing blood-based biomarkers of CAD, as it lies closest to the atherosclerotic plaque phenotype and may represent the net result of genetic factors and environmental influence. Cheng et al. described branched-chain amino acids, other hydrophobic amino acids, tryptophan breakdown products and nucleotide metabolites associated with cardiovascular disease [17]. Instead of atherosclerosis, Bodi et al. have used myocardial ischemia as phenotype to identify metabolic signatures [18]. Furthermore, metabolomics signatures of insulin resistance have been discovered using such an unbiased approach and have been introduced into clinical practice [19].

Since lipids appear to be causative in atherosclerotic CAD, several small studies have been published evaluating lipidomic signatures, using mostly clinical definitions of cardiovascular disease, rather than precision phenotyping for atherosclerosis. Meikle et al. identified lipidomic signatures that were different in clinically defined cohorts of stable versus unstable CAD [20]. In addition, there has been increasing interest in the role of ceramides as causative in atherosclerosis, as well as potential biomarkers for the prediction of adverse cardiovascular events [21]. The previously described GLOBAL study is significant in that it combines WGS, peripheral blood-based wholetranscriptome sequencing, unbiased proteomics, metabolomics and lipidomics in a single, large, prospective, precisely phenotyped patient cohort.

This population-based multi-omic approach also has the potential to also transform not only the drug target discovery paradigm by providing novel, more validated targets, but can also dramatically improve cycle time to bring medications to the market, instead of the current 14-16-year cycle and approximate US $\$ 2.2$ billion investment per new molecular entity to enter the market. We envision that in the future, there may be a more targeted, shorter regulatory path to prove safety in humans, but efficacy will be tested in a 'real-world setting', where a group of patients with a certain illness on a specific genetic background will be given the 'experimental' medication, and serial blood draws and phenotypic evaluations will be utilized to track gene expression, protein expression, metabolomics and other blood-based signatures, as well as phenotypic responses to therapy. Information gained from this multi-omic 'surveillance' will allow for real biologic evaluation of new molecular entities by studying how they perturb entire biological networks and affect literally thousands of molecules, rather than a single surrogate biomarker, such as LDL cholesterol.

\section{“...serial 'multi-omic' population-based surveillance in the real world will replace current randomized controlled trials and will become the new paradigm for pharmaceutical studies..."}

In conclusion, current approaches to risk stratification, early detection and therapy are inadequate today. For cardiovascular diseases, traditional risk assessment tools such as the FRS and the recently introduced global risk stratification tools fall short of the expectations of 21 st century citizens. Instead, by bringing forth bestin-class, cutting-edge scientific tools for novel scientific discovery, medicine, patient care and ultimately, our society, will be transformed. We believe that a comprehensive approach of precision phenotyping, pan-omics and systems biology-driven bioinformatics will lead to the discovery of novel biomarkers and therapeutic targets for not only atherosclerotic CAD, but many other disease states. After the introduction of such biomarkers for patient selection for specific therapy and proving the safety of novel compounds, serial 'multi-omic' population-based surveillance in the real world will replace current randomized controlled trials and will become the new paradigm for pharmaceutical studies, and will lead to the prolongation of a healthy, productive life.

\section{Financial \& competing interests disclosure}

$S$ Voros is founder, shareholder, employee and Chief Executive Officer of Global Genomics Group, LLC. He is also Executive Vice President and Chief Clinical Strategy Officer, and employee of Health Diagnostic Laboratory, Inc. The author has no other relevant affiliations or financial involvement with any organization or entity with a financial interest in or financial conflict with the subject matter or materials discussed in the manuscript apart from those disclosed.

No writing assistance was utilized in the production of this manuscript. 


\section{References}

1 Cheng VY, Berman DS, Rozanski A et al. Performance of the traditional age, sex, and angina typicality-based approach for estimating pretest probability of angiographically significant coronary artery disease in patients undergoing coronary computed tomographic angiography: results from the multinational coronary CT angiography evaluation for clinical outcomes: an international multicenter registry (CONFIRM). Circulation 124, 2423-2428 (2011).

2 Yeboah J, McClelland RL, Polonsky TS et al. Comparison of novel risk markers for improvement in cardiovascular risk assessment in intermediate-risk individuals. JAMA 308, 788-795 (2012).

3 Stone NJ, Robinson J, Lichtenstein AH et al. 2013 ACC/AHA Guideline on the Treatment of Blood Cholesterol to Reduce Atherosclerotic Cardiovascular Risk in Adults. A Report of the American College of Cardiology/American Heart Association Task Force on Practice Guidelines. J. Am. Coll. Cardiol. pii: S0735-1097(13)06028-2 (2013).

4 Drozdov I, Didangelos A, Yin X et al. Gene network and proteomic analyses of cardiac responses to pathological and physiological stress. Circ. Cardiovasc. Genet. 6, 588-597 (2013).

5 GLOBAL Clinical Study. http://clinicaltrials.gov/show/NCT01738828

6 Voros S, Rinehart S, Qian Z et al. Coronary atherosclerosis imaging by coronary CT angiography: current status, correlation with intravascular interrogation and metaanalysis. JACC Cardiovasc. Imaging 4, 537-548 (2011).

7 Voros S, Rinehart S, Qian Z et al. Prospective validation of standardized, 3-dimensional, quantitative coronary computed tomographic plaque measurements using radiofrequency backscatter intravascular ultrasound as reference standard in intermediate coronary arterial lesions: results from the ATLANTA (Asessment of Tissue Characteristics, Lesion Morphology, and Hemodynamics by Angiography with Fractional Flow Reserve, Intravascular Ultrasound and Virtual Histology, and Noninvasive Computed Tomography in Atherosclerotic Plaques) I study. JACC Cardiovasc. Interv. 4, 198-208 (2011).

8 Voros S, Joshi P, Qian Z et al. Apoprotein B, small-dense LDL and impaired HDL remodeling is associated with larger plaque burden and more noncalcified plaque as assessed by coronary CT angiography and intravascular ultrasound with radiofrequency backscatter: results from the ATLANTA I study. J. Am. Heart Assoc. 2, e000344 (2013).

9 Voros S, Rinehart S, Vazquez-Figueroa JG et al. Prospective, head-to-head comparison of quantitative coronary angiography, quantitative computed tomography angiography, and intravascular ultrasound for the prediction of hemodynamic significance in intermediate and severe lesions, using fractional flow reserve as reference standard (from the ATLANTA I and II study). Am. J. Cardiol. 113, 23-29 (2014).
10 Deloukas P, Kanoni S, Willenborg C et al. Large-scale association analysis identifies new risk loci for coronary artery disease. Nat. Genet. 45, 25-33 (2013).

11 Rosenberg S, Elashoff MR, Beineke P et al. Multicenter validation of the diagnostic accuracy of a blood-based gene expression test for assessing obstructive coronary artery disease in nondiabetic patients. Ann. Intern. Med. 153, 425-434 (2010).

12 Rosenberg S, Elashoff MR, Lieu HD et al. Whole blood gene expression testing for coronary artery disease in nondiabetic patients: major adverse cardiovascular events and interventions in the PREDICT trial. J. Cardiovasc. Transl. Res. 5, 366-374 (2012).

13 Thomas GS, Voros S, McPherson JA et al. A blood-based gene expression test for obstructive coronary artery disease tested in symptomatic nondiabetic patients referred for myocardial perfusion imaging. The COMPASS study. Circ. Cardiovasc. Genet. 6, 154-162 (2013).

14 Joshi PH, Rinehart S, Vazquez G et al. A peripheral blood gene expression score is associated with plaque volume and phenotype by intravascular ultrasound with radiofrequency backscatter analysis: results from the ATLANTA study. Cardiovasc. Diagn. Ther. 3, 5-14 (2013).

15 Voros S, Elashoff MR, Wingrove JA, Budoff MJ, Thomas GS, Rosenberg S, A peripheral blood gene expression score is associated with atherosclerotic plaque burden and stenosis by cardiovascular CT-angiography: results from the PREDICT and COMPASS studies. Atherosclerosis 233(1), 284-290 (2014).

16 Huan T, Zhang B, Wang Z et al. A systems biology framework identifies molecular underpinnings of coronary heart disease. Arterioscler. Thromb. Vasc. Biol. 33, 1427-1434 (2013).

17 Cheng S, Rhee EP, Larson MG et al. Metabolite profiling identifies pathways associated with metabolic risk in humans/clinical perspective. Circulation 125, 2222-2231 (2012).

18 Bodi V, Sanchis J, Morales JM et al. Metabolomic profile of human myocardial ischemia by nuclear magnetic resonance spectroscopy of peripheral blood serum: a translational study based on transient coronary occlusion models. J. Am. Coll. Cardiol. 59, 1629-1641 (2012).

19 Gall WE, Beebe K, Lawton KA et al. Alphahydroxybutyrate is an early biomarker of insulin resistance and glucose intolerance in a nondiabetic population. PLoS ONE 5, e10883 (2010)

20 Meikle PJ, Wong G, Tsorotes D et al. Plasma lipidomic analysis of stable and unstable coronary artery disease. Arterioscler. Thromb. Vasc. Biol. 31, 2723-2732 (2011).

21 Tarasov K, Ekroos K, Suoniemi M et al. Molecular lipids identify cardiovascular risk and are efficiently lowered by simvastatin and PCSK9 deficiency. J. Clin. Endocrinol. Metab. 99, E45-E52 (2014). 